\title{
Ultrasonic Phased Array Device for Acoustic Imaging in Air
}

\author{
Sevan Harput, and Ayhan Bozkurt, Member, IEEE
}

\begin{abstract}
An ultrasonic phased array device is developed to provide mobility aid for visually impaired people. To perform acoustic imaging, two different linear transducer arrays are constructed using commercially available transducers. The transmitter and receiver arrays are formed with six and four transducer elements, respectively. Individual transducer elements are discrete components with a radius of 1.9 wavelengths and a half-power beamwidth of $43^{\circ}$ at $40.8 \mathrm{kHz}$ center frequency. The transmitter array is formed by aligning the transducers with minimum spacing between the elements. Even this placement leads to the occurrence of unwanted grating lobes in the array response and decreases the Field of View to $30^{\circ}$. To eliminate these grating lobes, the elements of the receiver array are placed with a different spacing. Forming the receiver and transmitter arrays with non-identical element spacing makes the grating lobes to appear at different places. Since the response of the overall system is the product of the directivity patterns of receiver and transmitter arrays, the grating lobes diminish for the overall system and the Field of View increases.
\end{abstract}

Index Terms-Phased arrays, acoustic imaging, mobility aid for blind, ultrasonic obstacle detector.

\section{INTRODUCTION}

A COUSTIC imaging technology is widely used for medical purposes, underwater imaging and nondestructive testing applications [1]. In the past few decades acoustic imaging in air became popular with advances in air coupled transducers [2]. This led to the introduction of several electronic travel aid devices to improve the mobility of visually impaired people. Early devices which were in practical use include the Pathsounder [3], Sonic torch [4], Mowat sensor [5], Sonic pathfinder [6] and Nottingham Obstacle Detector [7]. These are also called obstacle detectors or path indicators, because they do not give guidance but only indicate the presence of an obstacle.

Among recently developed electronic travel aid devices, "Navbelt" and "GuideCane" get the highest attention. The "Navbelt", which was introduced by Shoval, Borenstein and Koren in 1994 [8], consists of a belt equipped with ultrasonic sensors and a small computer worn as a backpack. In 1997, the "GuideCane" was introduced by Borenstein and Ulrich [9]. Similar to the Navbelt, GuideCane uses an array of ultrasound sensors but it is relatively small, light, and easy to use. The working principle of both devices is based on array structure; without scanning the environment, each transducer element collects data from a single angle [10].

In 2002, a pocket PC based system was introduced by Choudhury, Aguerrevere and Barreto [11]. The device consists

A. Bozkurt is a faculty member in Sabanci University. of a SRF04 ultrasonic range sensors, a digital compass, a PIC16xx microcontroller and a counter for measuring the timing. The system transmits an acoustic pulse and measures the time of flight for range detection. In 2004, another device for detecting hanging objects was introduced by Debnath, Hailani, Jamaludin and Aljunid [12]. This device also uses the SRF04 Ranger kit for obstacle detection. The distance of the object is measured and converted to discrete levels of one, two and three meters. The SRF04 Ranger has the same ultrasonic transducers used in this work [13].

Phased array principles have not been extensively applied for acoustic imaging in air. A recent device that uses the phased array technique is an ultrasonic obstacle detector introduced by Strakowski, Kosmowski, Kowalik, and Wierzba in 2006 [14]. The developed device only performs receive beamforming by using a single ultrasound source and an array of microphones.

In this work, we present the design and experimental verification of an ultrasonic phased array device for acoustic imaging in air. The device is composed of two separate linear transmit and receive arrays and uses commercially available transducers. The transmit and receive arrays use the phase beamforming technique [15] to electronically sweep the acoustic beam and produce a sector scan for the detection of proximate objects [16], as shown in Fig. 1.

The intended application of the device is giving mobility aid to visually impaired people. Therefore, compactness and low power consumption are important design criteria. The device is built using 6 transmitter and 4 receiver elements and has an angular resolution of $4.23^{\circ}$. The sampling angle is selected as $4^{\circ}$ hence the acoustic beam is directed to 11 distinct angles from $-20^{\circ}$ to $20^{\circ}$. In order to eliminate the effect of grating lobes, the inter-element spacing is set to $2 \lambda$ and $3 \lambda$ for the transmitter and receiver arrays, respectively. This placement strategy increases the Field of View (FOV) beyond the halfpower beamwidth of individual transducer elements.

\section{BACKGROUND INFORMATION}

The operational principle of an acoustic phased array is identical to that of a phased array antenna. The only difference is in the form of the radiated energy: while the latter uses electromagnetic waves, the former radiates and detects pressure waves. The radiation pattern of the array is shaped by changing the relative phases of each array element. The 


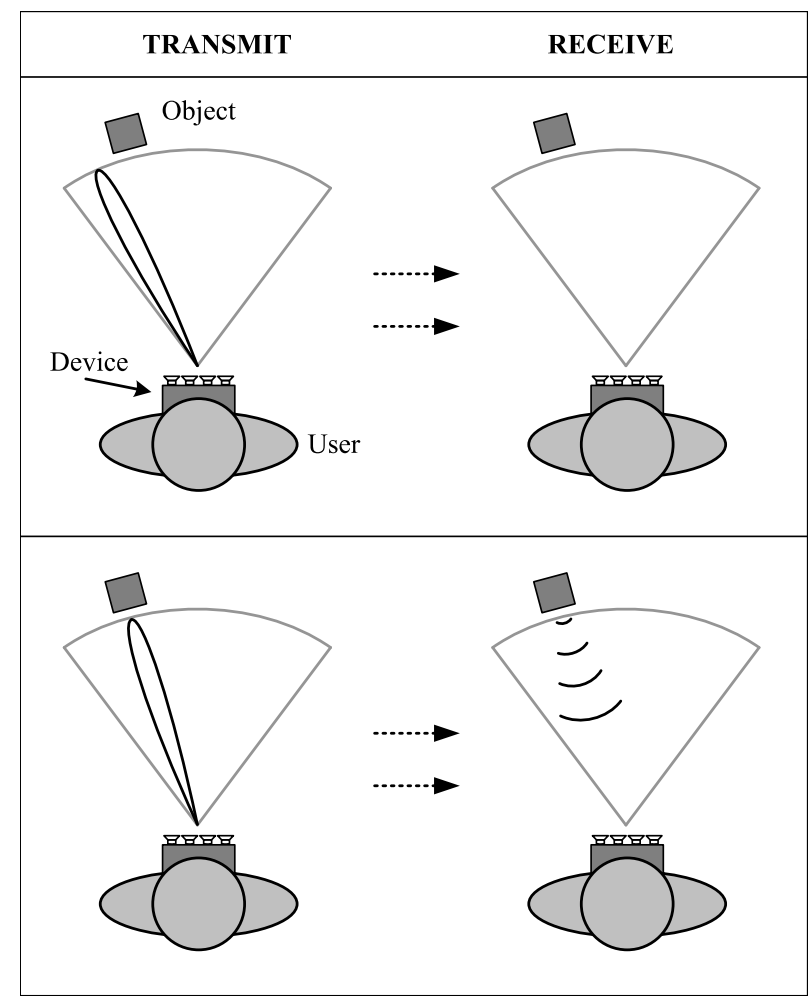

Fig. 1. Device scans the environment by transmitting ultrasonic beams in different angles and receiving reflecting echoes from objects

constructive and destructive interference among the signals radiated by the individual elements determine the effective radiation pattern of the array, which can be reinforced in a desired direction and suppressed in undesired directions [17].

The proposed system performs imaging by determining the acoustic reflectivity or opacity of objects in an acoustically transparent medium. To scan the beam across the imaging area, all elements of the phased array are driven with a variable phase or time delay [18]. The phase delays are controlled by software running on a microcontroller. The following section describes how the array pattern and phase delays are determined.

\section{A. Array Factor}

The array factor $(A F)$ is the directivity pattern of an array formed by omni-directional radiating transducer elements. The array factor for a uniform array can be calculated by summing the contributions of each element [19]. The normalized array factor is:

$$
A F_{n}=\frac{1}{N}\left[\frac{\sin \left(\frac{N}{2} \psi\right)}{\sin \left(\frac{1}{2} \psi\right)}\right]
$$

where $\psi$ is the relative phase between the array elements, and is defined as

$$
\psi=k d \sin \theta+\beta .
$$

Here $d$ is the spacing between the elements, $\theta$ is the observation angle in the far-field, $N$ is the number of the elements in the array, $\beta$ is the phase delay between consecutive array

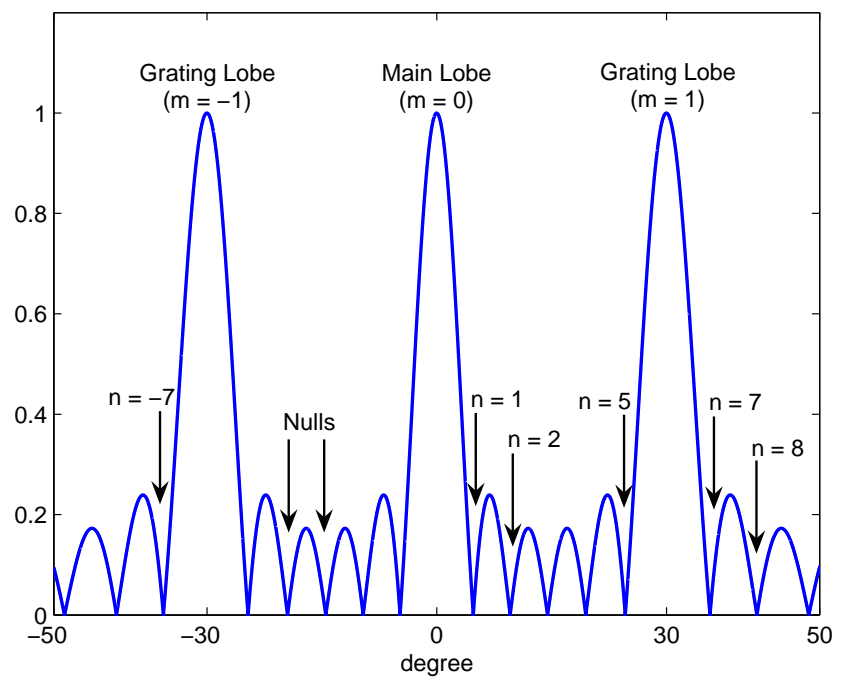

Fig. 2. Normalized array factor for a uniform array with 6 elements, $d=2 \lambda$ and $\beta=0^{\circ}$.

elements, and $k$ is the wave number. Detailed derivation of the array factor is shown in the appendix.

According to (1), the nulls of the array are found as

$$
\theta_{n}=\sin ^{-1}\left[\frac{1}{k d}\left(-\beta+\frac{2 n \pi}{N}\right)\right],
$$

for $n= \pm 1,2,3 \ldots$ and $n \neq N, 2 N, 3 N \ldots$.

Similarly, the maximum values appear at

$$
\theta_{m}=\sin ^{-1}\left[\frac{1}{k d}(-\beta+2 m \pi)\right], m= \pm 0,1,2 \ldots
$$

where $m=0$ corresponds to the main beam. The nulls and maxima of the normalized array factor (1) is shown in Fig. 2.

For a phased array, maximum radiation can be directed towards the beamsteering angle $\theta_{s}$ by equalizing the relative phase $\psi$ to zero:

$$
\begin{aligned}
\psi & =k d \sin \theta_{s}+\beta=0 \\
\beta & =-k d \sin \theta_{s}
\end{aligned}
$$

The phase delay between consecutive array elements for a particular beamsteering angle can be calculated using (4). Fig. 3 shows the normalized array factor for an array with 6 elements, $d=2 \lambda$ and $\theta_{s}=0^{\circ}$.

\section{B. Element Factor and Array Directivity Pattern}

The derivation of the array factor in (1) assumes that individual array elements are point sources. When the array is constructed using finite exciting sources, the element factor (the directivity pattern of the single element) has to be taken into account. The element factor is determined by the element dimensions, presence and shape of focusing layer, and, for the case of air-coupled transducers, by the size and shape of the casing and protective cage. In this work, 400ST/R160 standard open type transducers by Pro-Wave Electronic Corporation (Chung Ho City, Taipei Hsien, Taiwan) are used [20]. Table I lists the device parameters. Fig. 4 shows the element factor 
TABLE I

TRANSDUCER PARAMETERS

\begin{tabular}{ll}
\hline MANUFACTURER & Pro-Wave Electronic Corp., Taiwan. \\
TYPE & $400 \mathrm{ST} / \mathrm{R} 160$ (piezoelectric) \\
FREQUENCY & $40.8 \mathrm{kHz}$ \\
PHYSICAL SIZE & $16 \mathrm{~mm}(1.9 \lambda)$ \\
HP BEAMWIDTH & $43^{\circ}$ \\
\hline
\end{tabular}

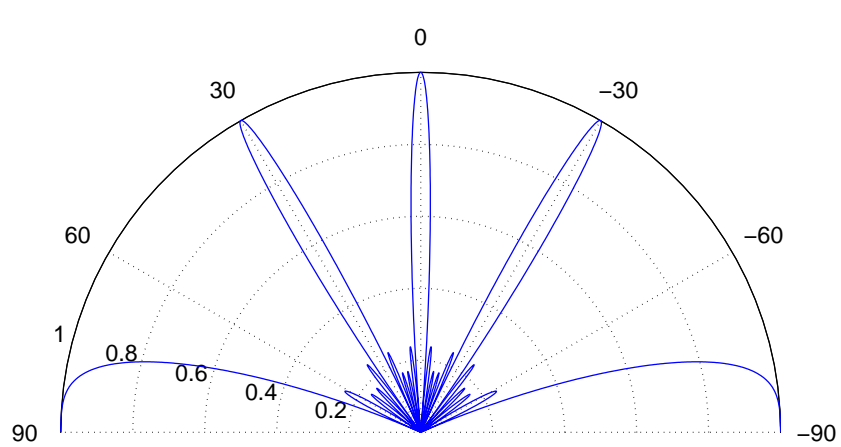

Fig. 3. Polar plot of the normalized array factor for a uniform array with 6 elements, $d=2 \lambda$ and $\theta_{s}=0^{\circ}$.

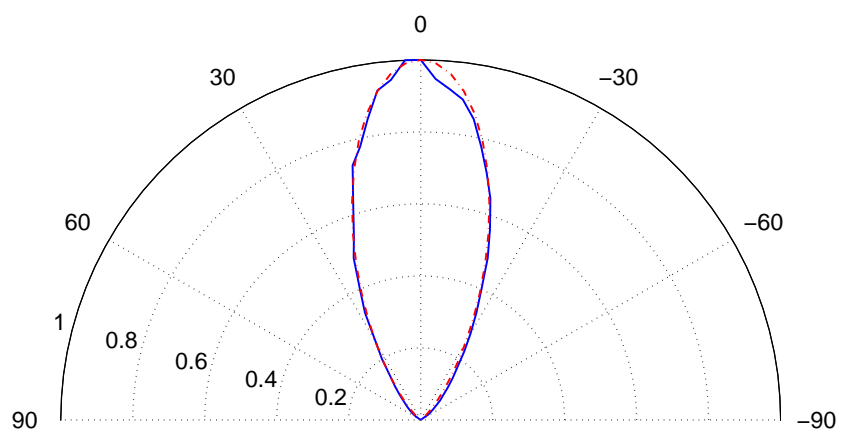

Fig. 4. Directivity pattern of single transducer: experimental (solid) and analytically fitted (dashed). Sidelobes beyond $\pm 60^{\circ}$ are omitted, since they are out of the active imaging area.

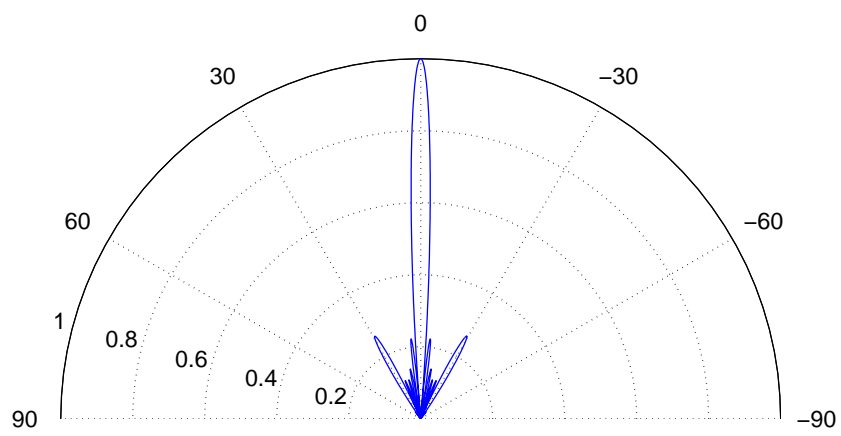

Fig. 5. The transmit radiation pattern of the array is the product of the element and array factors. The array is formed with 6 elements, $d=2 \lambda$ and $\theta_{s}=0^{\circ}$.

provided by the manufacturer together with an analytically fitted curve which is used in calculations.

The directivity pattern of the array (Fig. 5) is the product of the array factor (Fig. 3) and the element factor (Fig. 4) [19].

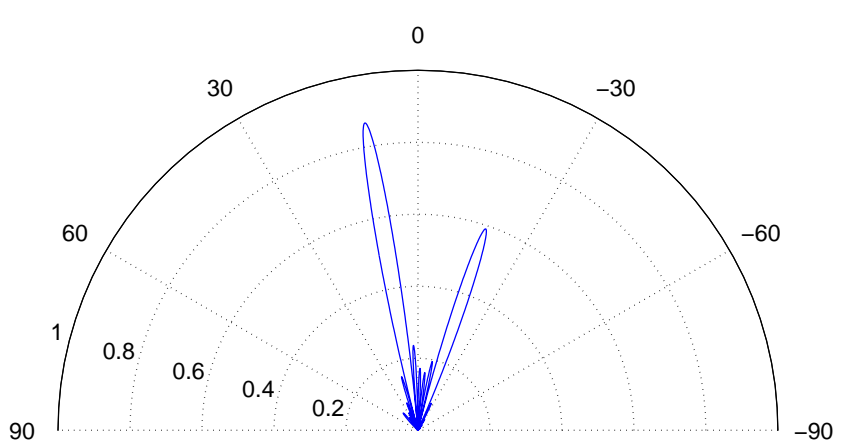

Fig. 6. Directivity pattern of a uniform array with 6 elements and $d=2 \lambda$. For $\theta_{s}=10^{\circ}$ the first grating lobe appears at $-20^{\circ}$. The amplitude of this lobe is smaller than the main beam due to the element factor.

\section{Methodology}

Imaging quality and system complexity are conflicting design parameters. By increasing the number of elements in an array better and sharper directivity patterns can be achieved, but this increases the complexity of the system and power consumption. In order to achieve a reasonable image quality, an angular resolution of less than $5^{\circ}$ is targeted. The angular resolution is determined by the half-power beamwidth of the main beam. For $N=5$ and $N=6$, the half-power beamwidth is found as $5.18^{\circ}$ and $4.23^{\circ}$, respectively. Consequently, the minimum number of array elements that satisfy the angular resolution condition is found as 6 . These calculations assume that $\theta_{s}=0^{\circ}$.

After constructing the transmitter array with 6 elements and $2 \lambda$ inter-element spacing, the directivity measurements show that element spacing larger than the Nyquist distance causes grating lobes inside the imaging area. If identical receiver and transmitter arrays were used, the FOV would be limited with $30^{\circ}$, which is the angle between the main beam and the first grating lobe. However, the selected transducer elements can be used for a scanning range of $43^{\circ}$ (Table I), which is larger than the $30^{\circ}$ limit for the FOV imposed by the array configuration. This limitation can be alleviated by applying different placement strategies for the receiver and transmitter arrays.

\section{A. Placement Strategy}

The transmit radiation pattern of a 6 element uniform array with $d=2 \lambda$ is shown in Fig. 6 . The grating lobe appears at $-20^{\circ}$ for a beamsteering angle of $10^{\circ}$. As this grating lobe is one of the maximum points in the array response other than the main beam, the location of the first grating lobe is found by solving the $\theta_{m}$ in (3) for $m= \pm 1$. For this array configuration, the grating lobe follows the main beam with nearly $30^{\circ}$. When the array is focused to $15^{\circ}$, the grating lobe appears approximately at $-15^{\circ}$. Scanning beyond the boundary of $\pm 15^{\circ}$ will result in ghost images if the same array configuration is used for receiving. For this reason, the receiver array is formed with a different placement strategy.

The effect of the first grating lobe in the transmitter array response can be reduced by adjusting the inter-element spacing 


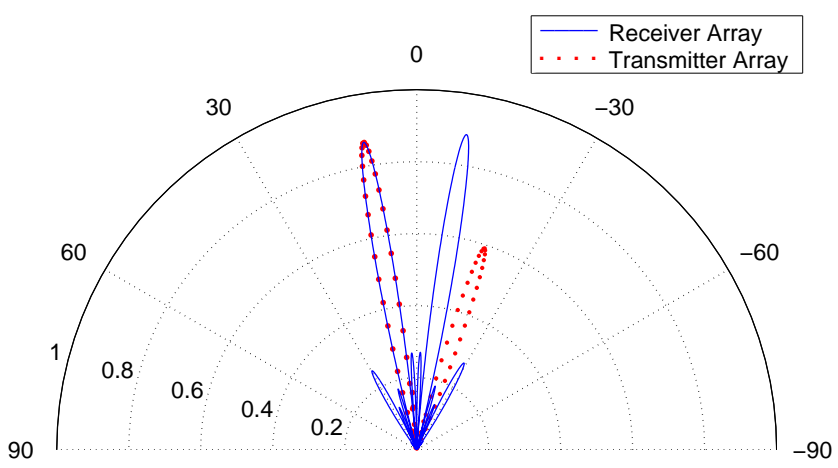

Fig. 7. Directivity patterns of the transmitter and receiver arrays for $\theta_{s}=10^{\circ}$. Transmitter array is formed with 6 elements and $d=2 \lambda$. Receiver array is formed with 4 elements and $d=3 \lambda$.

of the receiver array so that this grating lobe is aligned with one of the nulls of the receiver array response. The spacing between receiver array elements is found by equating $\theta_{m}$ of (3) for the transmitter array to $\theta_{n}$ of (2) for the receiver array.

$$
\begin{aligned}
\theta_{m} & =\sin ^{-1}\left[\frac{1}{k d_{t x}}\left(-\beta_{t x}+2 \pi\right)\right], \text { for } m=1 \\
\theta_{n} & =\sin ^{-1}\left[\frac{1}{k d_{r x}}\left(-\beta_{r x}+\frac{2 n \pi}{N_{r x}}\right)\right], n= \pm 1,2,3, \ldots
\end{aligned}
$$

For $\theta_{m}=\theta_{n}$, the receiver element spacing $d_{r x}$ is found as:

$$
\begin{aligned}
\frac{1}{k d_{t x}}\left(-\beta_{t x}+2 \pi\right) & =\frac{1}{k d_{r x}}\left(-\beta_{r x}+\frac{2 n \pi}{N_{r x}}\right) \\
\frac{d_{r x}}{d_{t x}} & =\frac{n}{N_{r x}}
\end{aligned}
$$

The array factor of the receiver has $N_{r x}-1$ nulls between consecutive maxima. Therefore, values of $n$ that would place the transmitter's grating lobe to a null which is farthest from both peaks is $n=(1 / 2+k) N_{r x}$ where $k=0,1,2, \ldots$. The solution for $k=0$ is not feasible as this results in $d_{r x}=d_{t x} / 2=\lambda$, which requires a spacing smaller than the transducer diameter. Solving (5) for $k=1$, we find the smallest realizable spacing as $d_{r x}=1.5 d_{t x}=3 \lambda$.

By using a spacing of $2 \lambda$, the FOV of the overall system can not be larger than $\pm 15^{\circ}$ with identical receiver and transmitter arrays. Setting $d_{r x}=3 \lambda$ moves the first grating lobe of the overall system to $\pm 30^{\circ}$ and increases the FOV to $60^{\circ}$. This value exceeds the limit imposed by the half-power beamwidth of an individual transducer element and makes the grating lobes to appear outside of the active imaging area.

Number of elements of the receiver array is determined by the angular resolution requirement. The half-power beamwidth of the main lobe is a function of the aperture size, $D=(N-1) d$. Consequently,

$$
\left(N_{r x}-1\right) d_{r x}=\left(N_{t x}-1\right) d_{t x},
$$

for which the nearest integer solution is $N_{r x}=4$.

Fig. 7 shows the transmit and receive directivity patterns for $\theta_{s}=10^{\circ}$. The directivity pattern of the overall system in Fig. 8 is the product of the directivity patterns of the transmitter and receiver arrays. This is also referred to as pattern multiplication for arrays of identical elements [19].

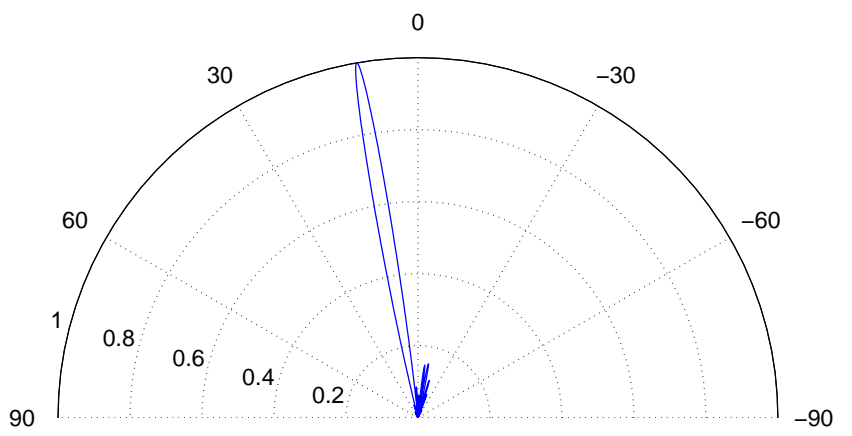

Fig. 8. Directivity Pattern of overall system $(\mathrm{TX} \times \mathrm{RX})$ for $\theta_{s}=10^{\circ}$.

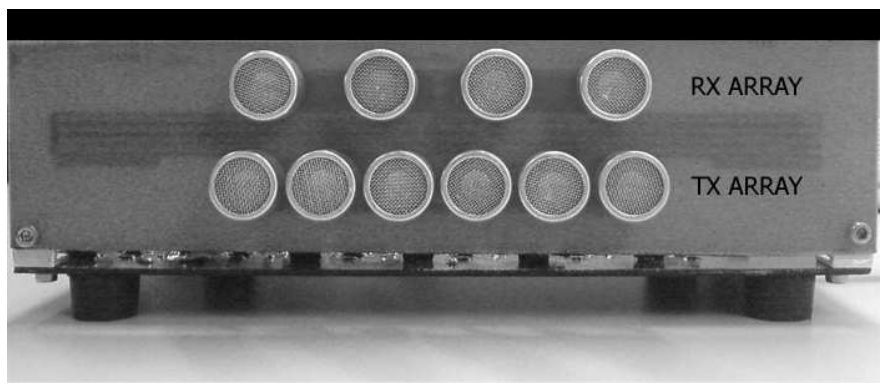

Fig. 9. Picture of the developed phased array device showing the transmitter and receiver arrays.

Pattern multiplication causes the grating lobes of transmitter and receiver array to disappear, consequently increasing the FOV.

After analyzing the directivity patterns above, the receiver and transmitter arrays are constructed with the mentioned placement strategy. Two linear arrays are realized by using the transducers with the features shown in Table I. Fig. 9 shows a picture of the constructed arrays.

\section{Measurements}

In general, the directivity pattern of an acoustic transducer is measured in an anechoic chamber. In this work, the measurements are done in a sufficiently large room, where no external noise source exists at the working frequency of transducers. For testing the performance of the system, the directivity patterns of the transmitter and receiver arrays are measured. The directivity pattern of the overall system is calculated by multiplying the measured array responses.

Fig. 10 shows the transmit radiation pattern of the ultrasonic phased array device. The dashed line is the theoretical array response and the solid line shows the measured directivity pattern of the transmitter array for a beamsteering angle of $16^{\circ}$. As expected, a grating lobe appears approximately at $-14^{\circ}$, whose amplitude is even larger than the main beam.

For measuring the directivity pattern of the receiver array, a single transmitter element is placed in front of the receiver array and receive beamforming is performed. In Fig. 11, the 


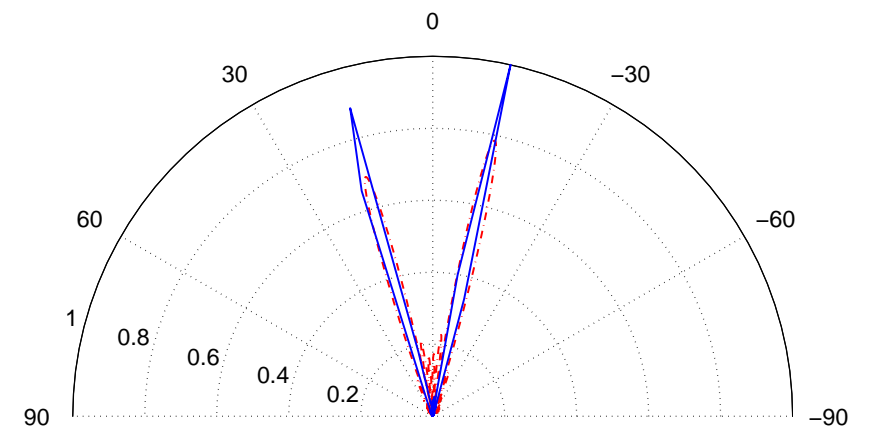

Fig. 10. Directivity pattern of transmitter array: measured (solid) and simulated (dashed). The transmitter array is formed with 6 elements, has a spacing of $d=2 \lambda$ and focused to $\theta_{s}=16^{\circ}$. A grating lobe appears at $-14^{\circ}$.

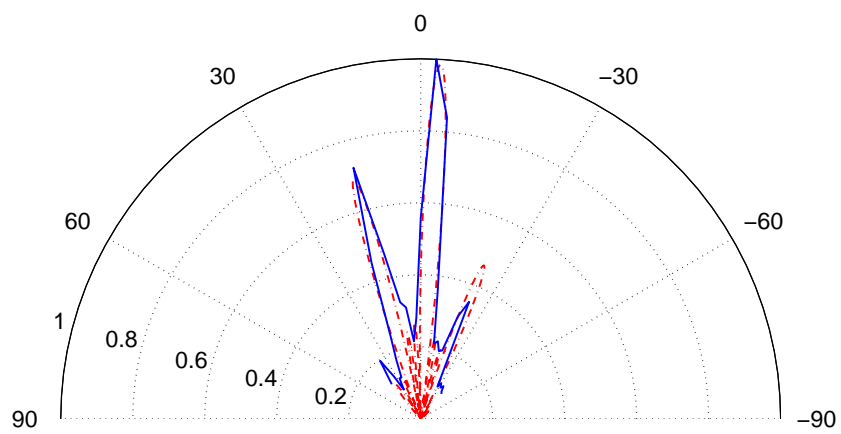

Fig. 11. Directivity pattern of receiver array: measured (solid) and simulated (dashed). The receiver array is formed with 4 elements, has a spacing of $d=3 \lambda$ and beamforming is performed for $\theta_{s}=16^{\circ}$. Two grating lobes appear at $-4^{\circ}$ and $-24^{\circ}$.

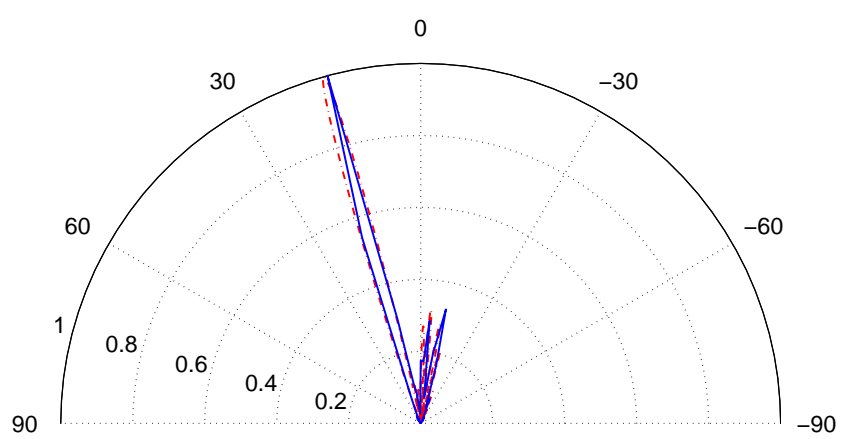

Fig. 12. Directivity pattern of overall system for $\theta_{s}=16^{\circ}$ : measured (solid) and simulated (dashed). The directivity pattern of the system is calculated by multiplying the measured directivity patterns of transmitter and receiver array.

dashed line shows the theoretical array response, and the solid line shows the measured directivity pattern of the receiver array, for a beamforming angle of $16^{\circ}$. For the receiver array, grating lobes appear approximately at $-4^{\circ}$ and $-24^{\circ}$.

Finally, the directivity pattern of overall system is found by multiplying the directivity patterns of transmitter array (Fig. 10) and receiver array (Fig. 11), which is shown in Fig. 12.
TABLE II

EXPERIMENTAL PARAMETERS

\begin{tabular}{ll}
\hline PARAMETER & VAlue \\
\hline SCANNING RANGE & $0.2 \mathrm{~m}$ to $3.5 \mathrm{~m}$ \\
FIELD OF VIEW & $40^{\circ}$ \\
Distal RESOLUTION & $\Delta r \approx 3.35 \mathrm{~cm}(4 \lambda)$ \\
SAMPLING ANGLE & $\Delta \theta=4^{\circ}$ \\
WORKING FREQUENCY & $\mathrm{f}=40.8 \mathrm{kHz}$ \\
ADC SAMPLING SPEED & $600 \mathrm{kSps}$ \\
PULSE WidTH & $8 \mathrm{cycles}(196 \mu \mathrm{sec})$ \\
\hline
\end{tabular}

$$
\begin{aligned}
& r=1.50 \mathrm{~m} \\
& \theta=-14^{\circ}
\end{aligned}
$$
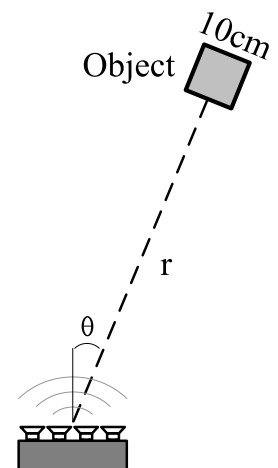

Device

Fig. 13. Experiment-I Setup

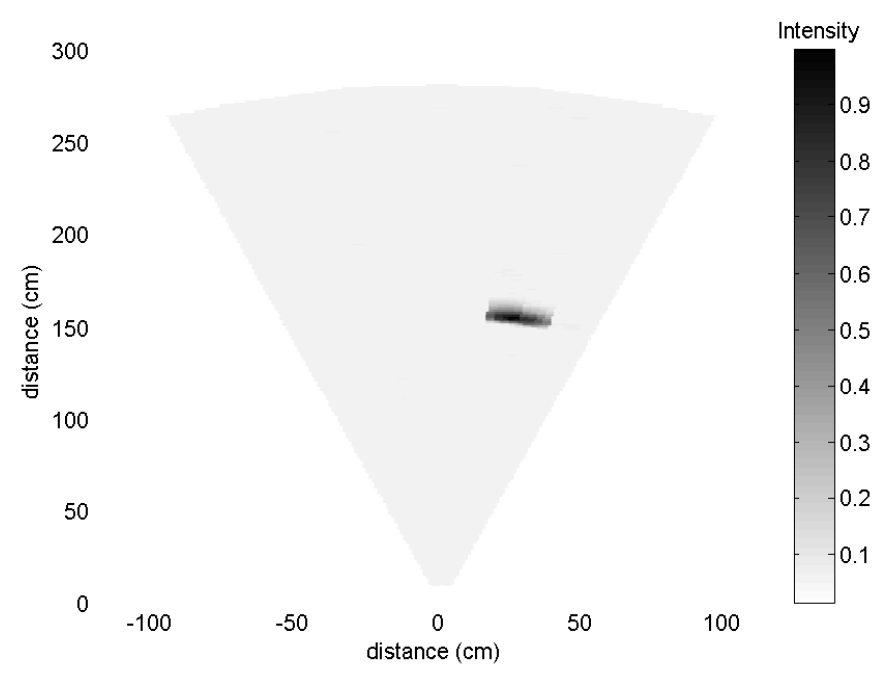

Fig. 14. Experiment-I Result

\section{EXPERIMENTS}

To measure the performance of the developed phased array device, experiments are performed on a set of objects with various shapes placed at different locations. The processor used in the developed device is an ADuC7024, an ARM based microcontroller produced by Analog Devices, Inc. Identical device settings and firmware code are used in all of the experiments. Beamsteering is performed in a range of $-20^{\circ}$ to $20^{\circ}$. The sampled echo by the ADC is transferred to a personal computer via an RS-232 link. Receive beamforming is done by using Matlab (The MathWorks Inc.) in $1^{\circ}$ angular steps. The image is formed by first converting the polar beamforming data 


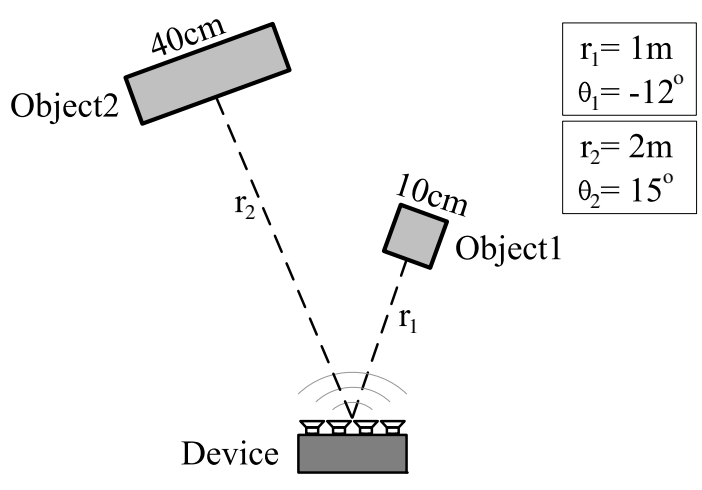

Fig. 15. Experiment-II Setup

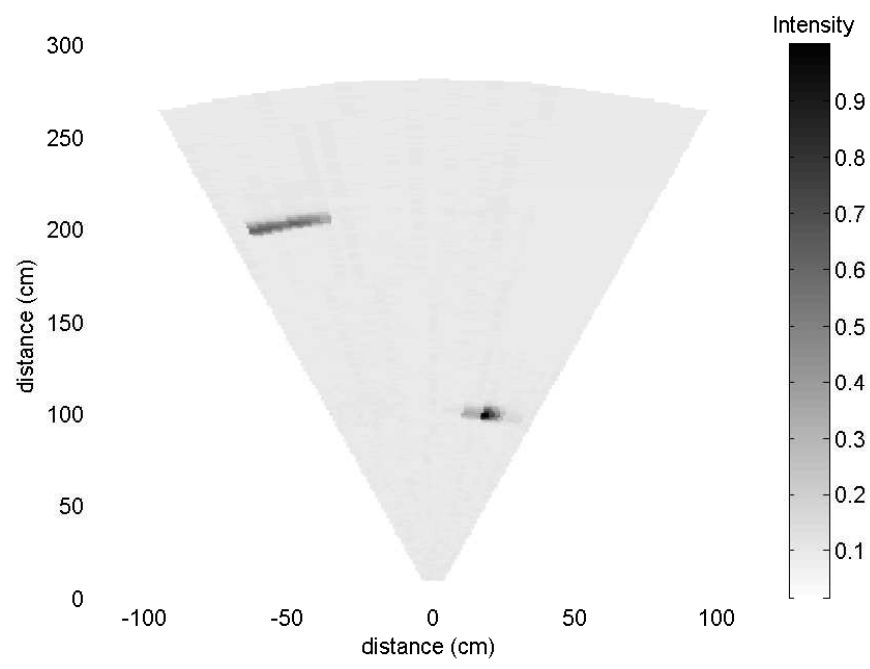

Fig. 16. Experiment-II Result

to Cartesian coordinates and then finding the missing pixels by interpolation. In all of experimental results, the device is at coordinate $(0,0)$ of the image.

\section{A. Experiment I}

In this experiment, a $10 \mathrm{~cm}$ wide wooden block is placed $150 \mathrm{~cm}$ away from the device at an angle of $-14^{\circ}$, as shown in Fig. 13. If identical receiver and transmitter arrays with an inter-element spacing of $2 \lambda$ were used, a grating lobe would appear at $-14^{\circ}$ for a beamsteering angle of $16^{\circ}$. This would result in a ghost object appearing at $16^{\circ}$ for this experimental setup. The result shown in Fig. 14 does not have any ghost objects. Consequently, this experiment proves that the presented placement strategy is successful in canceling the effects of grating lobes.

\section{B. Experiment II}

Two different wooden blocks are used in this experiment as shown in Fig. 15. A $10 \mathrm{~cm}$ wide block is placed $100 \mathrm{~cm}$ away from the device with an angle of $-12^{\circ}$. Another block of $40 \mathrm{~cm}$ width is placed at $15^{\circ}$ with a distance of $200 \mathrm{~cm}$. Both objects are detected successfully and they can be easily separated from each other as seen in Fig. 16. The observed

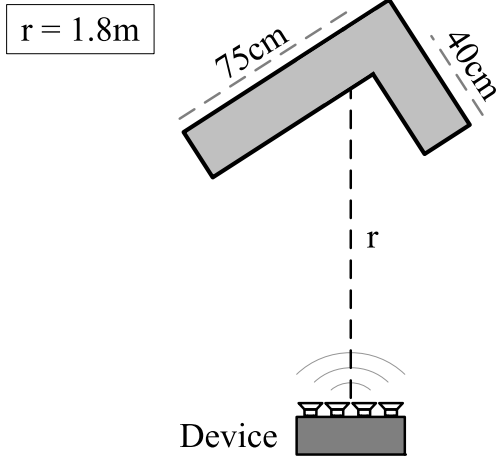

Fig. 17. Experiment-III Setup

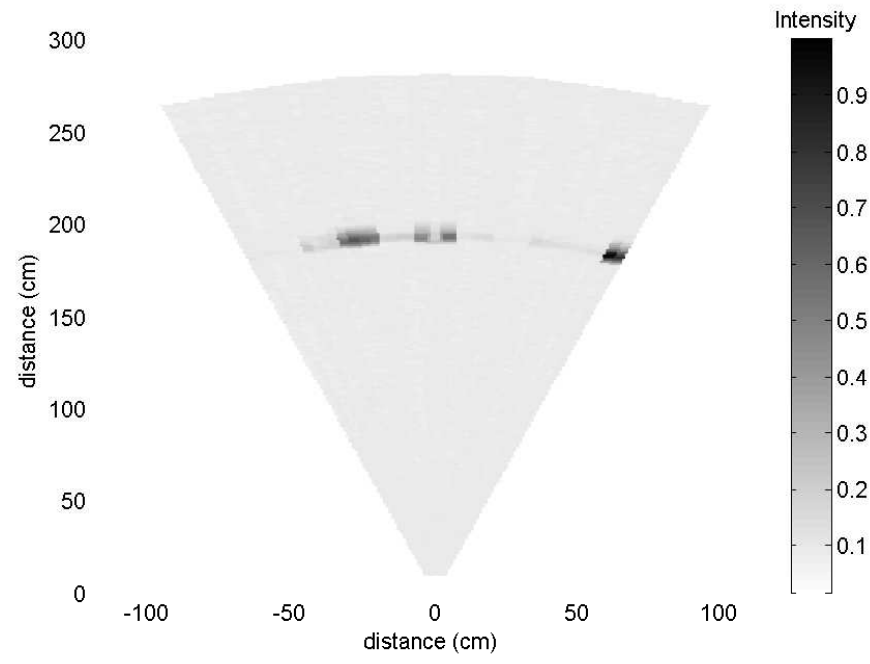

Fig. 18. Experiment-III Result

width of the object at $\mathrm{r}_{1}=1 \mathrm{~m}$ is approximately $18 \mathrm{~cm}$, so the object is widened by $4.5^{\circ}$. This value is within the angular resolution of the system. The object boundary is observed to have a thickness of $6.7 \mathrm{~cm}$ which corresponds the width of the transmitted pulse $(8 \lambda)$. The location of the real object can be estimated as the midpoint of the object in the image. This results in an error of $6.7 / 2=3.35 \mathrm{~cm}$, which is the distal resolution of the device. Again, no ghost objects are present in the image.

\section{Experiment III}

An L-shaped object is placed $180 \mathrm{~cm}$ away from the device, as shown in Fig. 17. The perpendicular sides of the L-shaped object form false-paths, due to which the exact shape of the object can not be determined. Although the image quality is poor, the collected object data can give the user enough information to avoid the obstacle (Fig. 18).

\section{Experiment IV}

A $3 \mathrm{~mm}$ wide plastic cable is hung $60 \mathrm{~cm}$ away from the device at an angle of $2.5^{\circ}$, as shown in Fig. 19. Plastic is a bad reflector and the cross-section of the object is quite small compared to the width of the main beam. This results in a 


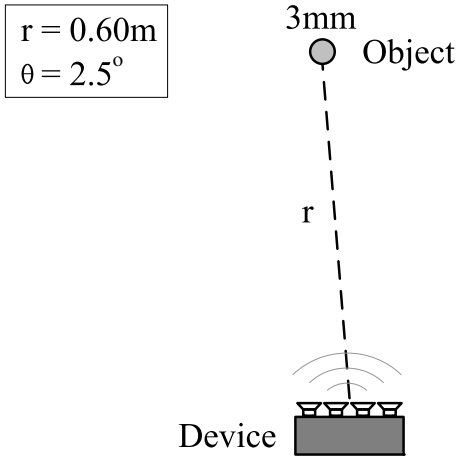

Fig. 19. Experiment-IV Setup

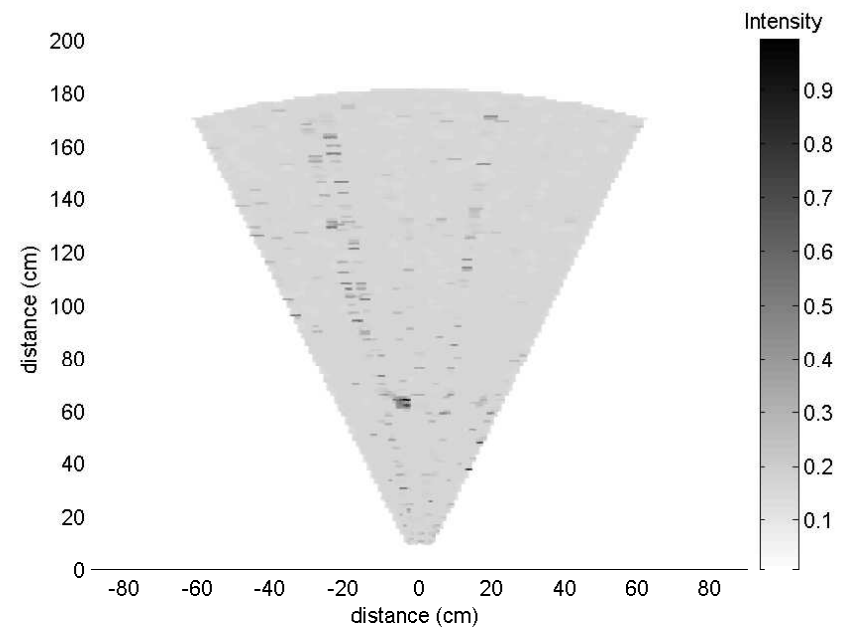

Fig. 20. Experiment-IV Result

significant degradation in the SNR. To improve the quality, the image in Fig. 20 is formed by pixel-wise adaptive Wiener filtering with neighborhoods of size $3 \times 3$.

\section{CONCLUSION}

In this work, we presented the design and experimental verification of a phased array device for ultrasonic imaging in air. The device was constructed using commercial transducers for ranging applications, which had a half-power beamwidth of $43^{\circ}$. As the dimension of the transducers were $1.9 \lambda$, the array response had grating lobes in an imaging area of $40^{\circ}$. This problem was solved by adjusting the inter-element spacing of the arrays. By forming the transmitter array with $2 \lambda$ and receiver array with $3 \lambda$ inter-element spacing, the first grating lobe was suppressed, and the FOV was extended to $60^{\circ}$.

We performed a set of experiments to verify the functionality of the device. Results prove that the applied placement strategy for the array elements is successful in suppressing the grating lobes as no ghost objects appear in the images. The angular and distal resolution specifications were also satisfied. The achieved imaging quality was sufficient to be used in a mobility aid device.

This research targeted the development of the imaging section of an electronic travel aid device. In order to have

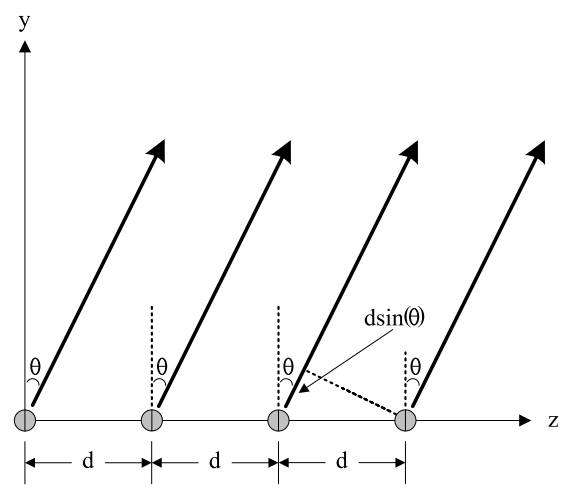

Fig. 21. $\mathrm{N}$ element array with uniform geometry

a complete system, the device must be combined with a user interface, such as an earphone or tactile display [21], [22], to convey the imaging data to the visually impaired person. The future research will focus on real-time imaging, since an offline imaging scheme is not practical for an electronic travel aid device.

The major advantage of the developed device is its ability to scan the imaging area using the phased array technique. An array to achieve the same angular resolution without scanning would require approximately the same number of transducers but individual elements have to be as wide as the aperture size of the phased array. Consequently, the developed phased array device is more compact than an array of transducers.

\section{APPENDIX \\ ARRAY FACTOR}

The array factor $(A F)$ is the directivity pattern of an array formed by omni-directional radiating transducer elements. $A F$ is a function of element spacing in wavelength, number of elements and phase delay between the elements. If the elements are spaced periodically and phased linearly with same amplitude, the array is called uniform. Fig. 21 shows a uniform array, where $d$ is the spacing between the elements and $\theta$ is the observation angle in the far-field. The array factor can be calculated by summing the contributions of each element:

$$
\begin{aligned}
A F & =1+e^{+j(k d \sin \theta+\beta)}+e^{+j 2(k d \sin \theta+\beta)}+\ldots \\
& +e^{+j(N-1)(k d \sin \theta+\beta)} \\
A F & =\sum_{n=1}^{N} e^{j(n-1)(k d \sin \theta+\beta)} .
\end{aligned}
$$

Here, $N$ is the number of the elements in the array, $\beta$ is the phase delay between consecutive array elements, and $k=$ $2 \pi / \lambda$. Relative phase between the elements, $\psi$, is defined as:

$$
\psi=k d \sin \theta+\beta .
$$


After simplification, $A F$ reduces to:

$$
\begin{aligned}
A F & =\frac{e^{j N \psi}-1}{e^{j \psi}-1} \\
& =e^{j[(N-1) / 2] \psi}\left(\frac{e^{j(N / 2) \psi}-e^{-j(N / 2) \psi}}{e^{j(1 / 2) \psi}-e^{-j(1 / 2) \psi}}\right) \\
& =e^{j[(N-1) / 2] \psi}\left(\frac{\sin \left(\frac{N}{2} \psi\right)}{\sin \left(\frac{1}{2} \psi\right)}\right)
\end{aligned}
$$

If the reference point is set to the physical center of the array, the array factor becomes:

$$
A F=\frac{\sin \left(\frac{N}{2} \psi\right)}{\sin \left(\frac{1}{2} \psi\right)}
$$

and the normalized array factor is

$$
A F_{n}=\frac{1}{N}\left[\frac{\sin \left(\frac{N}{2} \psi\right)}{\sin \left(\frac{1}{2} \psi\right)}\right] .
$$

The nulls of the array appear at

$$
\begin{gathered}
\sin \frac{N \psi}{2}=0 \Rightarrow \frac{N \psi}{2}=n \pi \quad, n= \pm 1,2,3 \ldots \\
N(k d \sin \theta+\beta)=2 n \pi \quad, n \neq N, 2 N, 3 N \ldots \\
\theta_{n}=\sin ^{-1}\left[\frac{1}{k d}\left(-\beta+\frac{2 n \pi}{N}\right)\right] .
\end{gathered}
$$

Similarly, the maximum values occur when

$$
\begin{gathered}
\frac{\psi}{2}=\frac{k d \sin \theta+\beta}{2}=m \pi \quad, m= \pm 0,1,2 \ldots \\
\theta_{m}=\sin ^{-1}\left[\frac{1}{k d}(-\beta+2 m \pi)\right] .
\end{gathered}
$$

\section{ACKNOWLEDGMENT}

The authors would like to thank Dr. Mustafa Karaman of Isik University, Istanbul, Turkey, for his guidance through the research and comments on the manuscript, and Dr. Meriç Özcan, and Mr. Yalçın Yamaner of Sabanci University, Istanbul, Turkey, for their valuable support during this work.

\section{REFERENCES}

[1] G. S. Kino, Acoustic Waves: Devices, Imaging, and Analog Signal Processing. Rentice-Hall, Inc., 2000.

[2] M. Haller and B. Khuri-Yakub, "1-3 composites for ultrasonic air transducers," in Proc. IEEE Ultrasonics Symposium, vol. 2, 1992, pp. 937-939.

[3] L. Russel, "Travel path sounder," in Proc. Rotterdam Mobility Research Conference. New York: Amer. Foudation for the Blind, 1965.

[4] L. Kay, "An ultrasonic sensing probe as a mobility aid for the blind," Ultrasonics, vol. 2, p. 53, 1964.

[5] N. Pressey, "Mowat sensor," Focus, vol. 3, pp. 35-39, 1977.

[6] A. Heyes, "Sonic pathfinder: a programmable guidance aid for the blind," Electronics and Wireless World, vol. 90, no. 1579, pp. 26-29,62, 1984.

[7] A. Dodds, J. Armstrong, and C. Shingledecker, "The nottingham obstacle detector: development and evaluation," Journal of Visual Impairment and Blindness, vol. 75, no. 5, pp. 203-209, 1981.
[8] S. Shoval, J. Borenstein, and Y. Koren, "Mobile robot obstacle avoidance in a computerized travel aid for the blind," in IEEE International Conference on Robotics and Automation, vol. 3, 1994, pp. 2023-2028.

[9] J. Borenstein and Y. Ulrich, "The guidecane-a computerized travel aid for the active guidance of blind pedestrians," in Proc. IEEE International Conference on Robotics and Automation, vol. 2, 1997, pp. 1283-1288.

[10] S. Shoval, J. Borenstein, and Y. Koren, "Navbelt and the guide-cane [obstacle-avoidance systems for the blind and visually impaired]," IEEE Robotics and Automation Magazine, vol. 10, no. 1, pp. 9-20, 2003.

[11] M. Choudhury, D. Aguerrevere, and A. Barreto, "A pocket-pc based navigational aid for blind individuals," in IEEE Symposium on Virtual Environments, Human-Computer Interfaces and Measurement Systems, 2004, pp. 43-48.

[12] N. Debnath, Z. Hailani, S. Jamaludin, and S. Aljunid, "An electronically guided walking stick for the blind," in Proc. of the 23rd Annual International Conference of the IEEE Engineering in Medicine and Biology Society, vol. 2, 2001, pp. 1377-1379.

[13] SRF04 - Ultra-Sonic Ranger, Devantech, 2003.

[14] M. Strakowski, B. Kosmowski, R. Kowalik, and P. Wierzba, "An ultrasonic obstacle detector based on phase beamforming principles," IEEE Sensors Journal, vol. 6, no. 1, pp. 179-186, 2006.

[15] O. T. V. Ramm and S. W. Smith, "Beam steering with linear arrays," IEEE Transactions on Biomedical Engineering, vol. BME-30, no. 8, pp. 438-452, 1983.

[16] K. Higuchi, K. Suzuki, and H. Tanigawa, "Ultrasonic phased array transducer for acoustic imaging in air," in IEEE Ultrasonics Symposium, 1986, pp. 559-562.

[17] G. of Telecommunication Terms, Federal Standard 1037C, 1996.

[18] R. Mailloux, Phased Array Antenna Handbook, 2nd ed. Artech House, 2005.

[19] C. A. Balanis, Antenna Theory, 3rd ed. Wiley-Interscience, 1997, ch. Arrays: Linear, Planar, and Circular, pp. 283-320.

[20] 400ST/R160 Air Ultrasonic Ceramic Transducer, Pro-Wave Electronic Corp., 2005.

[21] M. Shinohara, Y. Shimizu, and A. Mochizuki, "Three-dimensional tactile display for the blind," IEEE Transactions on Rehabilitation Engineering, vol. 6, no. 3, pp. 249-256, 1998.

[22] Y. Ikei, K. Wakamatsu, and S. Fukuda, "Vibratory tactile display of image-based textures," IEEE Computer Graphics and Applications, vol. 17 , no. 6, pp. 53-61, 1997.

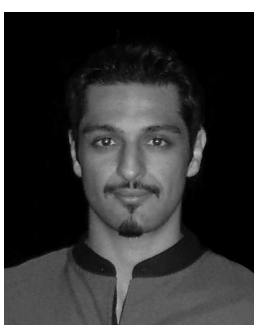

Sevan Harput received the B.Sc. degree in microelectronics engineering and the M.Sc. in electronic engineering and computer sciences from Sabanci University, Turkey in 2005 and 2007, respectively. Currently he is a research assistant in the microelectronics department. His research interests include biomedical instrumentation, medical imaging and ultrasound.

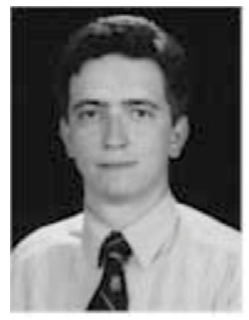

Ayhan Bozkurt received his B.Sc., M.Sc., and Ph.D degrees from Bilkent University in 1992, 1994, and 2000 , respectively, all in electrical and electronics engineering. He is currently working as an assistant professor on the Faculty of Engineering and Natural Sciences of Sabanci University, Istanbul, Turkey. His research interests are transducer modeling and fabrication, and integrated circuit design. Dr. Bozkurt is a member of IEEE. 